Methods 146 metabolites, including dietary-related lipoproteins, fatty acids, cholesterol and amino acids, were included. Partial least squares discriminatory analyses (PLSDA) and sparse PLSDA (sPLSDA) were used to distinguish ethnic-specific metabolite signatures of GDM in 2668 WEs and 2671 PK pregnant women (gestational age $\leq 196$ days) in the BIB cohort. The impact of BMI on the metabolome and GDM risk, along with other known GDM risk factors (age, parity, multiple pregnancy and smoking status), was also examined.

Results Seven metabolites across a panel of metabolic processes (fatty acids, glycolytic, and cholesterol metabolism) were found to be predictive of GDM in both ethnicities, with fatty acids appearing to be more important drivers of GDM within WEs. Additionally, 6 metabolites were predictive of GDM solely within WEs, whilst no distinct metabolite associations were observed in PKs. Following the stratification of women by their BMI, case-status and ethnicity, a distinct metabolite profile was identified within normal-weight PK cases when compared to all other cases, characterised by a panel of amino acids and cholesterols, and glycolytic and unsaturated fatty acid metabolites.

Conclusion Serum-metabolite profiles differ by ethnicity and GDM status, largely driven by differences in fatty acid and cholesterol metabolite levels. However, in normal-weight PK women, a broad range of metabolic processes are uniquely altered and offer insight into the elevated risk of GDM observed in this otherwise healthy population. Future investigations into the determinants of these differences in metabolite profiles may shed light on the aetiology of elevated GDM risk in healthy $\mathrm{PK}$ women and direct the development of more efficacious intervention strategies.

\section{P82 REHOUSING OLDER SOCIAL HOUSING TENANTS: HEALTH-RELATED PROCESSES AND OUTCOMES}

${ }^{1}$ Stefanie Buckner*, ${ }^{1}$ Calum Mattocks, ${ }^{2}$ Lindsay Blank. ${ }^{1}$ Cambridge Public Health, University of Cambridge, Cambridge, UK; ${ }^{2}$ School of Health and Related Research, University of Sheffield, Sheffield, UK

\subsection{6/jech-2021-SSMabstracts. 170}

Background In the context of a social housing crisis, the UK must meet the housing needs of an ageing population. Rehousing schemes run by social housing providers are important means to support older (aged 55+) social housing tenants to move to appropriate homes. A better understanding of how well these schemes work is needed. This paper is based on a study (01/2020-12/2021) of the health-related processes and outcomes linked to the rehousing of older social housing tenants in Hackney/London through four local authority-supported schemes: Downsizing; Regeneration; Housing Moves; Seaside \& Country Homes.

Methods A mixed methods design entailed a scoping review, based on a search of scientific databases (MEDLINE, EMBASE, Scopus, CINAHL, PsychINFO) and the grey literature, and a narrative synthesis of results. Interviews with practice-based stakeholders across sectors $(n=11)$ were conducted. A survey of older social housing tenants who have moved through a rehousing scheme $(n=766)$, analysed in Excel, generated descriptive statistics and themes for qualitative exploration. A Photovoice component with 16 survey participants, currently underway, has been designed to capture the participants' rehousing experience and its effect on their lives. It entails interviews and focus groups. Interviews and a focus group are being conducted with older social housing tenants who were interested in being rehoused, but opted against moving ('non-movers'; $n=5$ ). All qualitative data are analysed thematically in NVivo12. All methods have been adjusted to comply with Covid-19 rules.

Results The review yielded three peer-reviewed papers and 13 reports, thus revealing a gap in knowledge around the healthrelated effects of rehousing older social housing tenants. Interviews with practice-based stakeholders highlighted promising approaches in the rehousing schemes, such as highly personalised support, and challenges, including a lack of suitable homes for tenants to move into. The survey yielded 62 valid responses (8.1\%). As well as helpful elements (e.g. being given choices) and challenges (e.g. poor communication) in the rehousing process, it identified welcome (e.g. a warmer home) and unwelcome (e.g. loneliness) health and wellbeing outcomes. The findings will be broken down by rehousing scheme. They will be supplemented by detailed insights from the Photovoice components and data collection with nonmovers.

Conclusion The study was designed to generate insights that can inform efforts by Hackney Council and other social housing providers to improve rehousing schemes for older tenants and optimise their health-related effects. Channels through which findings will be shared with decision makers and the public include a national workshop and a photo exhibition.

\section{P83 ABSTRACT WITHDRAWN}

\section{P84 ABSTRACT WITHDRAWN}

\section{P85 HOW TO TACKLE UNINTENTIONAL DISCRIMINATION IN PRIMARY HEALTH CARE: GENERAL PRACTITIONERS' IMPLICIT BIASES AND CULTURAL COMPETENCE}

Camille Duveau*, Vincent Lorant. Institute of Research Health and Society, University Catholic of Louvain, Brussels, Belgium

\subsection{6/jech-2021-SSMabstracts. 171}

Background Reducing ethnic inequalities in health care is a worldwide priority. General practice is no exception to this, as studies pointed to ethnic discrimination in diagnosis, referral and treatment of racial minorities patients by General Practitioners (GPs). However, it is unclear whether these discriminations are due to implicit bias or by the lack of explicit cultural competences, two explanations demanding different intervention. Implicit biases are a human trait which can influence stereotypes and care behaviour whereas cultural competence is 'the ability to work and communicate effectively and appropriately with people from culturally different backgrounds'.

Methods This mixed-method study investigates the existence of implicit biases and the level of cultural competences among GPs trainees. Data collection among GPs trainees (2021) was used and analysed in SAS Enterprise Guide. The 170 